\title{
Clear evidence for element partitioning effects in a Ti-6Al-4V alloy by the first-principles phase field method
}

\author{
Thi Nu Pham ${ }^{1}$, Kaoru Ohno ${ }^{1}$, Ryoji Sahara ${ }^{2}$, Riichi Kuwahara \\ and Swastibrata Bhattacharyya ${ }^{4}$ \\ ${ }^{1}$ Department of Physics, Yokohama National University, 79-5 Tokiwadai, Yokohama \\ 240-8501, Japan \\ ${ }^{2}$ National Institute for Materials Science (NIMS), 1-2-1 Sengen, Tsukuba 305-0047, \\ Japan \\ ${ }^{3}$ Dassault Systèmes K.K., ThinkPark Tower, 2-1-1 Osaki, Shinagawa-ku, Tokyo \\ 141-6020, Japan \\ ${ }^{4}$ Department of Physics, Birla Institute of Technology and Science Pilani, K. K. Birla \\ Goa Campus, Zuarinagar, Goa 403726, India \\ E-mail: ohno@ynu.ac.jp
}

\begin{abstract}
Ti-6wt\%Al-4wt\%V (Ti64) is an $\alpha+\beta$ titanium alloy, in which the alloying components strongly affect the mechanical properties. In this report, element partitioning effects in Ti64 are investigated by using the first-principles phase field (FPPF) method, which has recently been proposed by our group. In the FPPF method, the local free energy is calculated using cluster expansion method in combination with density functional theory and the temperature effect is incorporated using potential renormalization theory. We have succeeded in identifying enrichment of $\mathrm{Al}(\mathrm{V})$ in the $\alpha(\beta)$ phase, i.e., the clear evidence for the element partitioning effects of $\mathrm{Al}$ and $\mathrm{V}$, without using any thermodynamical parameter. The transformation of the $\beta$ phase and the $\alpha$ phase in microstructure is investigated by varying the $\mathrm{V}$ and $\mathrm{Al}$ concentration by a small amount. Our results are in excellent agreement with the recent experimental results, showing the validity of the FPPF method for ternary alloys.
\end{abstract}

Keywords: First-principles phase field method, Ti64 alloy, element partitioning

\section{Introduction}

Nowadays Ti-6wt\%Al-4wt\% V (Ti64) is one of the most used Ti alloys in industry with a broad range from aerospace to medical applications. Ti64 is a two-phase Ti alloy, which has the hexagonal close packed structure $(\mathrm{HCP})(\alpha)$ and the body centered cubic structure $(\mathrm{BCC})(\beta)$. The $\alpha+\beta$ titanium shows high strength, relatively low density and good resistance against fatigue crack initiation and corrosion [1-5]. The mechanical properties and the properties related to phase transformations of Ti64 are strongly 
influenced by the microstructure, which is determined by the history of processing, thermal treatment procedures, and alloying element partitioning effects [6-8].

Element partitioning effects on the phase transformation and mechanical properties of Ti alloys have been studied very recently in both experiments and simulations [9-13]. It is known that $\mathrm{Al}$ and $\mathrm{O}$ exhibit as the $\alpha$ stabilizer and have a high concentration in the $\alpha$ phase. In contrast, the $\beta$ stabilizers such as $\mathrm{V}, \mathrm{Mo}, \mathrm{Cr}$, and Fe prefer to diffuse into the $\beta$ matrix [10]. The plasticity decreases, while the tensile strength and the yield strength increases if the $\mathrm{Al}$ or $\mathrm{V}$ concentration increases even by a very small amount [7]. By using a combination of scanning electron microscopy (SEM) and energy dispersive spectroscopy (EDS), Banerjee et al. [14] measured chemical compositions and microstructures across the graded Ti-8Al- $x \mathrm{~V}$. They have indicated that the volume fraction of the $\alpha$ phase decreases and the volume fraction of the $\beta$ phase increases as the $\mathrm{V}$ content increases. On the other hand, with increasing mass content of $\mathrm{Al}$, the $\beta$ grain size decreases as was reported by Tan et al. [15], who used laser solid forming (LSF). Very recently, Huang et al. studied the influence of thermal treatments on element partitioning effects in the $\alpha+\beta$ titanium alloy using both experiments and simulations [13]. They analyzed the effects of the solution temperature and the holding time on the alloying element partitioning by electron probe micro-analysis (EPMA), and observed quantitatively the $\mathrm{Al}$ and $\mathrm{V}$ distributions in the two phases and at the phase boundary. Then, they performed the phase field (PF) simulation to investigate the element partitioning during the $\alpha \rightarrow \beta$ transformation. In their PF simulation, the initial microstructure characterized by SEM was used as a direct input, and the free energy was formulated based on other thermodynamical parameters [13]. To the best of our knowledge, there is no direct first-principles-based simulation on element partitioning effects of Ti64 that does not rely on empirical parameters of the $\alpha$ and $\beta$ phases.

Traditionally, it is very costly and time-consuming to design $\mathrm{Ti}$ alloys and to model their processes [7]. One of the most challenging task in computational materials science is to predict and control precisely the evolution of material microstructures. The recent advance in computing technology helps scientists to simulate the microstructure of materials without consuming much time and cost. One can find some recent computational approaches to microstructure simulations in the paper by Zhang et al. [16], such as cellular automation [6,16,17], Monte Carlo [18,19], phase field (PF) [20-22], and crystal plasticity finite element $[23,24]$. However, it should be emphasized that all these methods have been applied only empirically. There are input parameters, for example, thermodynamic and kinetic data, which are difficult to obtain from experiments [16]. To overcome this problem, the first-principles phase field (FPPF) method was proposed by our group [25]. In the FPPF method, instead of using the continuous Ginzburg-Landau-type polynomial free energy as in almost all traditional PF methods, the step-wise local free energy is introduced to describe atomic arrangements and local compositions. This step-wise free local energy is calculated by combining density functional theory (DFT) [26,27], cluster expansion theory [28], and potential renormalization theory $[29,30]$. The method was applied to $\mathrm{Ni}-\mathrm{Al}$ alloys at $1027^{\circ} \mathrm{C}$ 
and succeeded in reproducing the evolution of microstructures as a function of only the average composition without using any thermodynamic empirical parameter [25].

In the present report, we extend the FPPF method to a ternary alloy, Ti64. By using the number densities of the constituent elements as the phase field variables, we can compute the distribution map of each element in an alloy without using any thermodynamic empirical parameter. Here we particularly focus on element partitioning effects of the Ti64 alloy and investigate the influence of systematically varying the $\mathrm{Al}$ and $\mathrm{V}$ concentrations by using the FPPF method. We compare our results with the recent experimental results.

\section{Methodology}

The detail of the FPPF method was explained in our previous paper [25]. In this method, we have considered number densities $\varphi_{X}$ of the constituent elements i.e., $X=$ $\mathrm{Ti}\left(\varphi_{N i}\right), \mathrm{Al}\left(\varphi_{A l}\right)$, and $\mathrm{V}\left(\varphi_{V}\right)$ as the phase field variables. The local free energy $F$ as a functional of these phase field variables was calculated by using potential renormalization theory $[29,30]$ together with cluster expansion theory $[28,31,32]$. Using Pipeline Pilot

and Materials Studio produced by Dassault Systemes, Inc., and the CAmbridge Serial Total Energy Package (CASTEP) [33] for DFT calculations, we developed a protocol that automatically submits a huge number of DFT calculations with CASTEP to find the step function of the local free energy $F$ in the crystal structures of FCC, BCC, and HCP phases, which are required as inputs for the FPPF simulations. The detailed design of the protocol is explained in Appendix. In principle, our method can be applicable to any kind of alloys, and here we demonstrate the extension of the theory from binary systems to ternary alloys. The explicit functional form for the local free energy can be represented as:

$$
F\left(\varphi_{T i}, \varphi_{A l}, \varphi_{V}\right)=\sum_{n, m, l}^{4} f_{n}\left(\varphi_{T i}\right) f_{m}\left(\varphi_{A l}\right) f_{l}\left(\varphi_{V}\right) E_{T i_{n} A l_{m} V_{l}},
$$

where $f_{i}(x)=\theta(x-i)-\theta(x-i-1)$ and $E_{T i_{n} A l_{m} V_{l}}$ is the renormalized energy of the $T i_{n} A l_{m} V_{l}$ cluster. In the cases of $(n+m+l)<0$ and $(n+m+l)>4$, the local free energy is positive and increases sharply by using a polynomial function to avoid unrealistic composition [25]. The chemical potentials are given by

$$
\begin{aligned}
& \mu_{\mathrm{Ti}}=F\left(\varphi_{\mathrm{Ti}}+0.5, \varphi_{\mathrm{Al}}, \varphi_{\mathrm{V}}\right)-F\left(\varphi_{\mathrm{Ti}}-0.5, \varphi_{\mathrm{Al}}, \varphi_{\mathrm{V}}\right)-\varepsilon_{\mathrm{Ti}} \nabla^{2} \varphi_{\mathrm{Ti}}, \\
& \mu_{\mathrm{Al}}=F\left(\varphi_{\mathrm{Ti}}, \varphi_{\mathrm{Al}}+0.5, \varphi_{\mathrm{V}}\right)-F\left(\varphi_{\mathrm{Ti}}, \varphi_{\mathrm{Al}}-0.5, \varphi_{\mathrm{V}}\right)-\varepsilon_{\mathrm{Al}} \nabla^{2} \varphi_{\mathrm{Al}}, \\
& \mu_{\mathrm{V}}=F\left(\varphi_{\mathrm{Ti}}, \varphi_{\mathrm{Al}}, \varphi_{\mathrm{V}}+0.5\right)-F\left(\varphi_{\mathrm{Ti}}, \varphi_{\mathrm{Al}}, \varphi_{\mathrm{V}}-0.5\right)-\varepsilon_{\mathrm{V}}, \nabla^{2} \varphi_{\mathrm{V}}
\end{aligned}
$$

where $\epsilon_{X}(\mathrm{X}=\mathrm{Ti}, \mathrm{Al}$ or $\mathrm{V})$ denotes the gradient energy coefficient of $\varphi_{\mathrm{X}}$, and $\epsilon_{X} \nabla^{2} \varphi_{\mathrm{X}}$ represents the interface energy contribution. For the conserved order parameters, such as the composition and the atomic density, the Cahn-Hilliard equation is applicable. The generalized Cahn-Hillard equation is derived from the continuity equation $\partial \varphi_{\mathrm{X}} / \partial t=$ 
$-\nabla \cdot \mathbf{J}_{X}$ and the flux, $\mathbf{J}_{X}=-M_{X} \nabla \mu_{X}$ :

$$
\frac{\partial \varphi_{\mathrm{X}}}{\partial t}=M_{X} \nabla^{2} \mu_{X} .
$$

For simplicity, we assume that $M_{X}$ and $\epsilon_{X}$ to be independent of the species X, and simulation time and simulation cell size to be arbitrary units, which can be scaled to the experimental time and length if required [25]. It is essentially important to incorporate the random force in the phase field equation, because the local free energy is reconstructed with its local values. The amplitude of the random force is chosen as 0.5 for all the calculations. The grid space of $156 \times 156$ is used, and the grid space $(d x)$, the time step $(d t)$ are set at 0.8 and 0.00001 , respectively. The PFFP simulations are performed for a time step until the microstructure becomes almost stable (typically around $10^{5}$ steps for Ti64). Because $\mathrm{V}$ has only a very small amount (less than $5 \mathrm{wt} \%$ ), we assume that the initial structure contains Ti- and Al-rich/poor seeds and V is distributed homogeneously.

\section{Results and discussion}

There are 34 possible atomic structures of $\operatorname{Ti}_{n} \mathrm{Al}_{m} \mathrm{~V}_{l}$ with $n+m+l \leq 4$; see Table. 1 . The renormalized energy in Eq. (1) is given by the sum of the total energy and the renormalization energy in Table. 1. We have performed the calculation for both BCC and $\mathrm{HCP}$ structures, and then compared the two local free energies. Because Ti is the main element in Ti64 (90wt\%), so we used the optimized lattice parameter for pure Ti alloys by CASTEP programs based on the generalized gradient approximation (GGA) using the PBE functional [34]. For Ti64, the cutoff energy is set at $500 \mathrm{eV}$, and the lattice parameter is $3.32 \AA$ for $\mathrm{BBC}$ unit cell, and $a=2.94, c=4.63 \AA$ for HCP unit cell. The experimental lattice parameter for $\mathrm{BBC}$ is $3.32 \AA$, and $a=2.95, c=4.68 \AA$ for $\mathrm{HCP}$ unit cell [35]. For some of the stoichiometric compositions, the assumed structures are similar to those treated in the previous first-principles calculations $\left(\mathrm{Ti}_{3} \mathrm{Al}\right.$ [36], $\mathrm{Ti}_{2} \mathrm{Al}_{2}$ [36,37], $\mathrm{TiAl}_{2}$ [36,37], $\mathrm{TiAl}_{3}[36,38], \mathrm{Ti}_{2} \mathrm{AlV}$ [39]). The structure that has smaller free energy is chosen for the input of the local free energy in the FPPF model. For example, the HCP structure was chosen for $\mathrm{Ti}_{4}$, and the BCC structure was chosen for $\mathrm{Ti}_{2} \mathrm{Al}_{2}$. In the case of pure $\mathrm{Ti}$, the $\mathrm{HCP}-\mathrm{BCC}$ transition temperature $\mathrm{T}_{c}$ is $2517^{\circ} \mathrm{C}$; see Fig.1. Even though this result is different from experimental data (the experimental $\mathrm{T}_{c}$ is $882^{\circ} \mathrm{C}$ [40]), it shows an improvement in comparison to the phonon calculation where $\mathrm{T}_{c}$ is calculated as $2727^{\circ} \mathrm{C}$ [41]. In this report, a simple version of potential renormalization theory is applied, where all atoms are fixed at the lattice points except for a central atom that is displaced around the lattice point, although a more precise calculation could be in principle done with the displacement of atoms at surrounding lattice points $[29,30]$. 


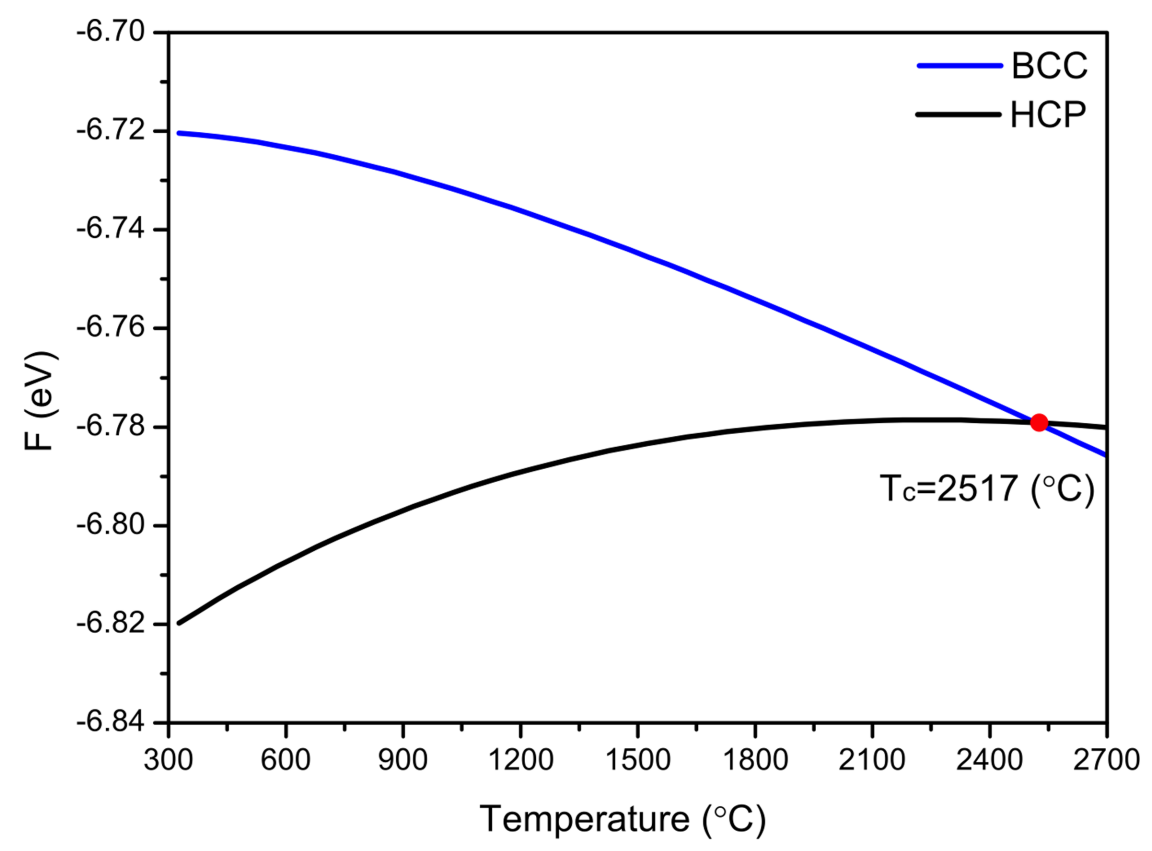

Figure 1: The local free energy corrections per basic tetrahedron (primitive unit cell including four atoms) of pure $\mathrm{Ti}$ in the $\mathrm{HCP}$ and $\mathrm{BCC}$ structures versus temperature.

The initial input energy/ local free energy was constructed from both HCP and BCC atomic structures. In this study, the interstitial atoms were not considered for simplicity, and the unit cells were assumed to have only $\mathrm{Ti}, \mathrm{Al}, \mathrm{V}$ and vacancies. 
Evidence for element partitioning effects in Ti64 by FPPF method

Table 1: Tetrahedral clusters with all possible non-equivalent combinations of $\mathrm{Ti}, \mathrm{Al}$, and $\mathrm{V}$, including vacancies. The total energies/renormalization energies, in $\mathrm{eV} /$ unit cell, calculated by first-principles DFT with GGA using CASTEP program is shown below for each structure. The renormalization energy at $980^{\circ} \mathrm{C}$ was calculated by using potential renormalization theory.

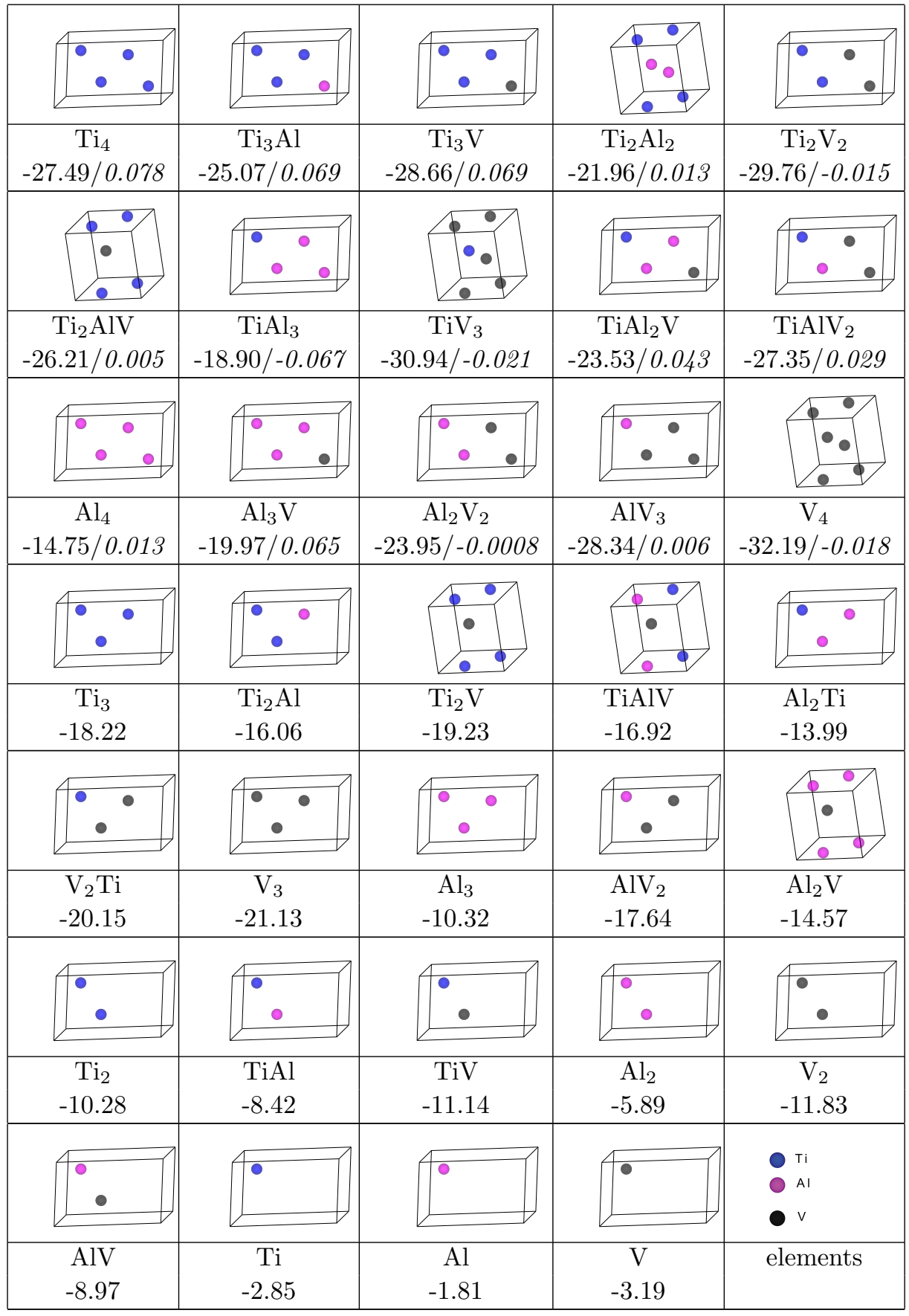


Fig.2 (a) and (b) are the distribution map of $\mathrm{Al}$ and V. Here, the color scale represents the element concentration, that increases from black to white. From this figure, one can see clear evidence about the enrichment of $\mathrm{Al}$ in the $\alpha$ phase and $\mathrm{V}$ in the $\beta$ phase, which clearly suggests element partitioning effects. Here, the element partitioning effects mean that $\mathrm{Al}$ and $\mathrm{V}$ are selectively partitioned into $\alpha$ and $\beta$ phase, respectively. That is, in these effects, $\mathrm{Al}$ prefers to populate in the $\alpha$ phase and $\mathrm{V}$ prefers to populate in the $\beta$ phase. Our results are in a good agreement with the experimental results of Huang et al. [13] (see also Fig.1 of their paper), who demonstrated that the $\alpha$ phase has higher $\mathrm{Al}$ concentration while the $\beta$ grain boundary has higher $\mathrm{V}$ concentration; see Fig.2 (c).
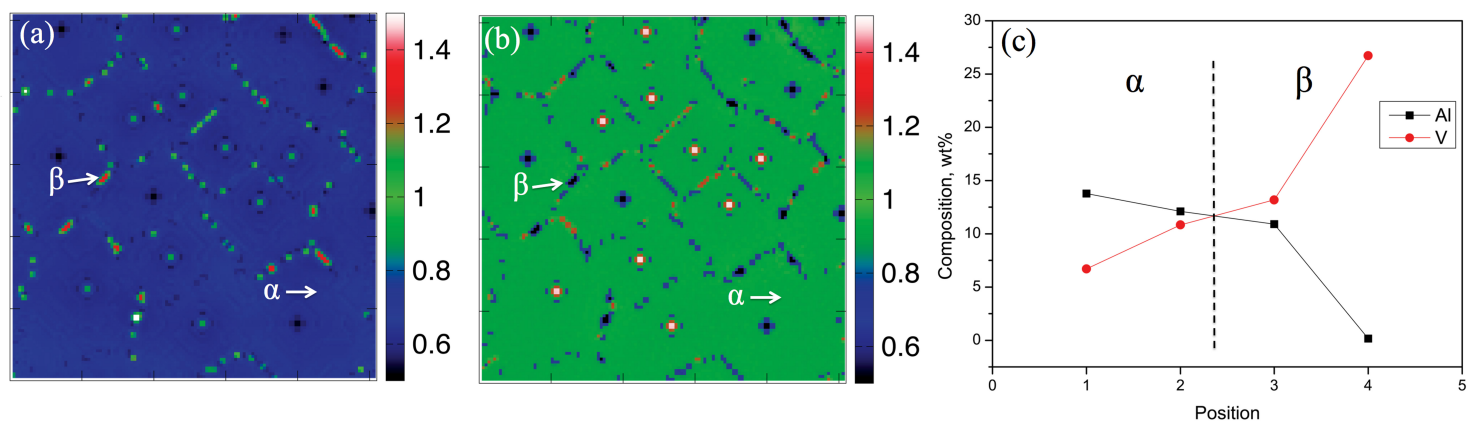

Figure 2: Plot of the number density distribution of (a) $\mathrm{V}$ and (b) Al at the simulation time $t=10$. (c) shows the values of $\mathrm{Al}$ and $\mathrm{V}$ concentration at four positions $1,2,3$, and 4 , where 1 -the center of $\alpha ; 2,3$-the edge of $\alpha, \beta$ near the $\alpha / \beta$ boundary, respectively; and 4 -the center of $\beta$.

The evolution of the microstructure during the simulation time is shown in Fig.3. There are several brighter points at time $t=10$ that transform to darker points at later times in the $\mathrm{Al}$ concentration figure; see Fig.3 (d), (e), and (f). These points represent the transformation from the $\alpha$ phase to the $\beta$ phase. This change is simply caused by the fact that the initial seeds of $\mathrm{Al}$ are the brighter points, which gradually diffuse in the $\alpha$ phase. 

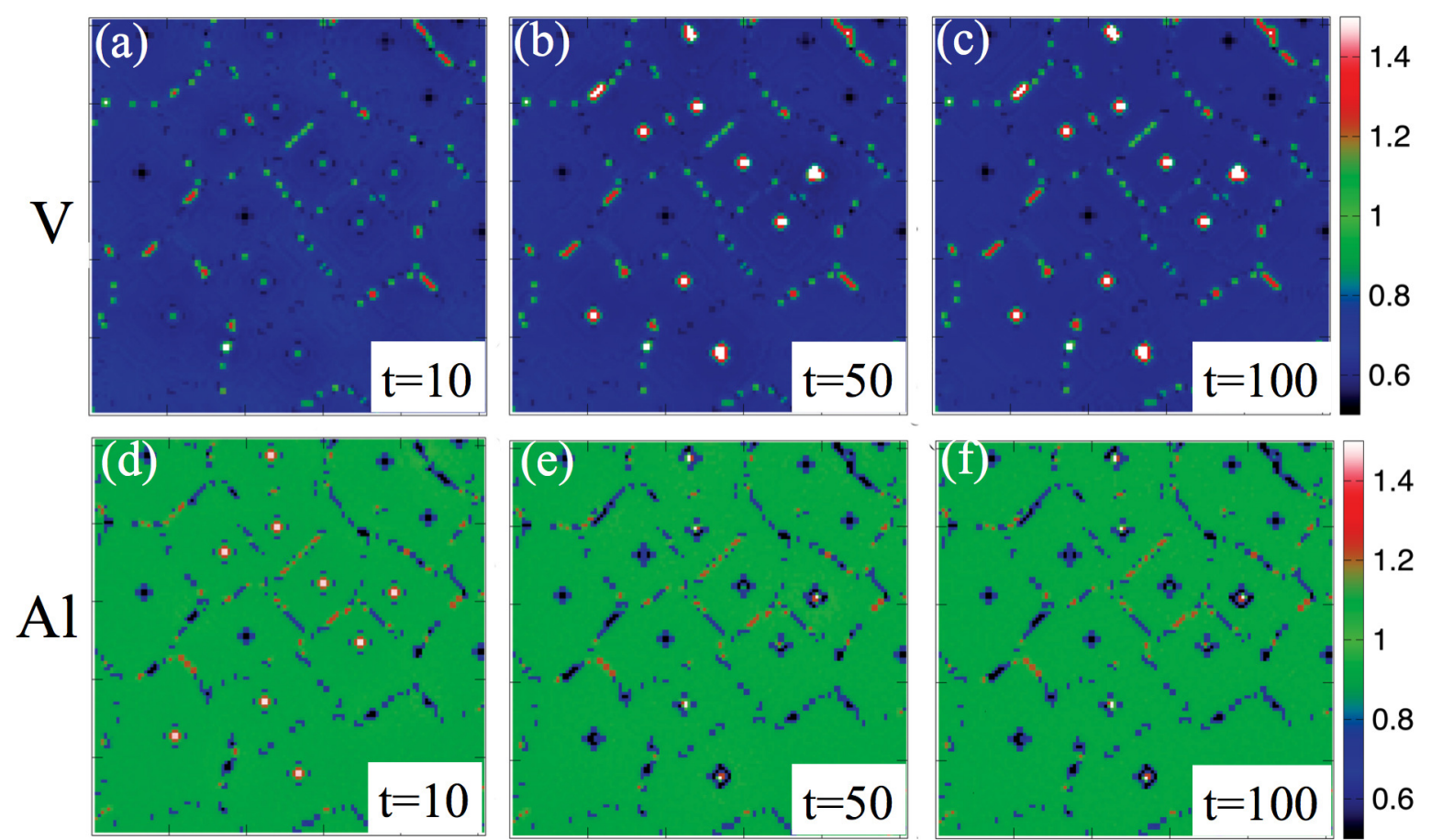

Figure 3: Time evolution of microstructures during the simulation. (a), (b), and (c) show the evolution of the V concentration, (d), (e), and (f) show the evolution of the Al concentration. The bright colour parts have higher concentration of the element than the dark colour parts.

Fig. 4 presents the time evolutions of the local free energy and the derivative of the local free energy. When the $\beta$ grain boundary and the $\alpha$ phase are stable, the local free energy has the minimum value. 

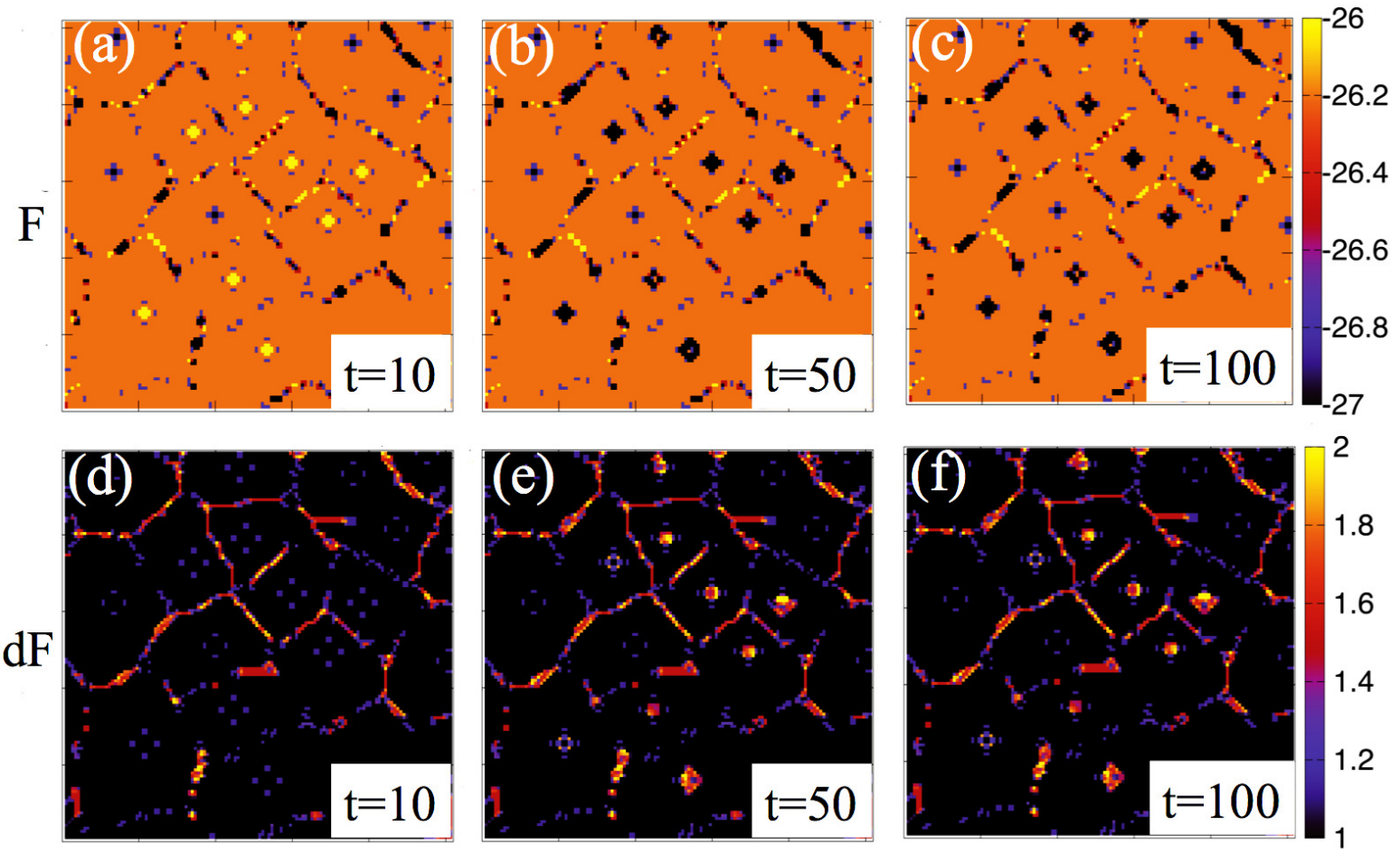

Figure 4: (a), (b), and (c) present the local free energy and (d), (e), and (f) present the derivative of the local free energy at $\mathrm{t}=10,50$, and 100 , respectively.

The local free energy has lower values and the derivative of the local free energy has higher values at the $\beta$ grain boundary. Our results are consistent with the study of Alabort et al. [42], who stated that the changes in the volume fraction of the $\beta$ phase are responsible for the transition from dislocation-creep to superplastic grain boundary sliding.

Next, the change in the $\beta$ grain boundary is investigated by modifying the $\mathrm{V}$ concentration by a very small amount. Here we keep the Al concentration at 6 wt $\%$ while increase $\mathrm{V}$ from $4.1 \mathrm{wt} \%$ to $5.2 \mathrm{wt} \%$. As the $\mathrm{V}$ concentration increases, there is a decrease in the volume fraction of the $\alpha$ phase and an increase of the $\beta$ phase. One can realize the $\beta$ grain boundary getting brighter and there are more points which have higher V concentration; see Fig.5 (a), (b), and (c) for V concentration and Fig.5 (d), (e), and (f) for Al concentration. These results are in a good agreement with the experimental results of Banerjee et al. [14]. 

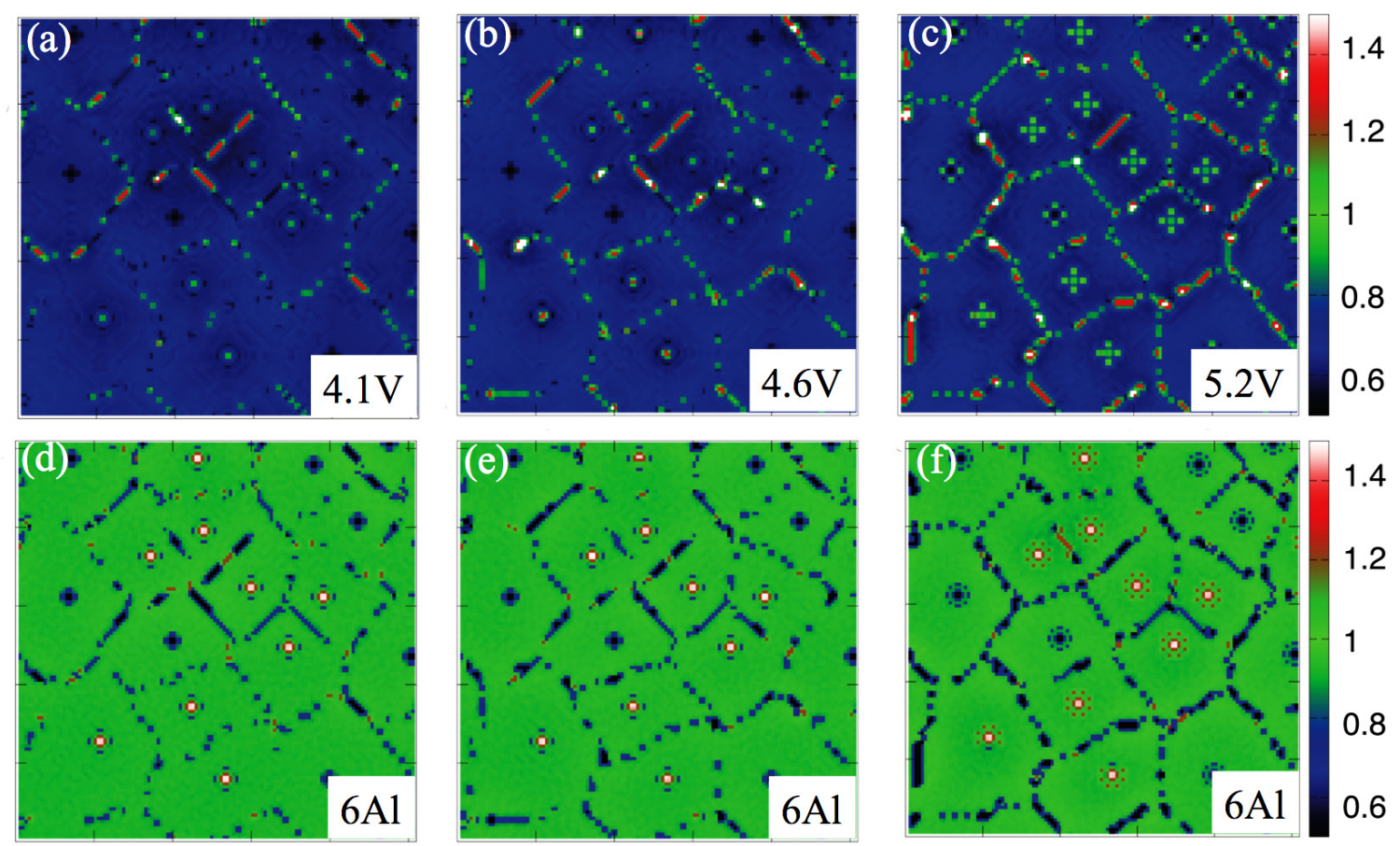

Figure 5: Change in the microstructure when the $\mathrm{V}$ concentration is increased from 4.1wt\% to $5.2 \mathrm{wt} \%$. (a), (b), and (c) are the $\mathrm{V}$ concentration while (d), (e), and (f) are the $\mathrm{Al}$ concentration.

On the other hand, the concentration of $\mathrm{V}$ is kept as $6 \mathrm{wt} \%$ while $\mathrm{Al}$ is increased from $6.1 \mathrm{wt} \%$ to $6.6 \mathrm{wt} \%$. The results are shown in Fig.6. The volume fraction of the $\alpha$ phase increases from Fig.6 (d) to Fig.6 (f), and there appear more brighter points, which is a good agreement with the experimental results of Tan et al. [15]. When Al concentration is increased with fixed $\mathrm{V}$ concentration, the volume fraction of the $\alpha$ phase increased because $\mathrm{Al}$ is selectively populated in the $\alpha$ phase due to the element partitioning effects. 

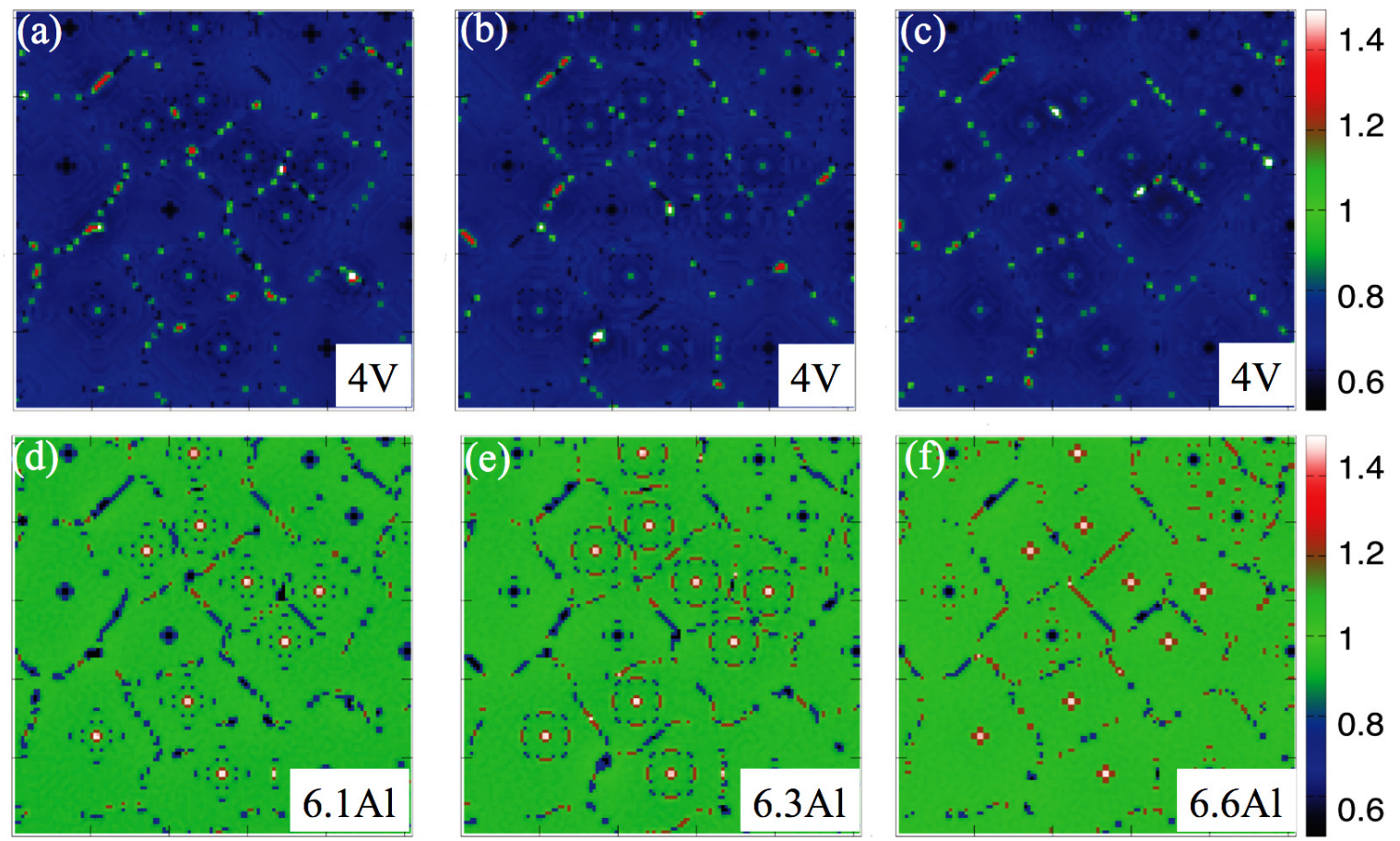

Figure 6: Change in the microstructure when the $\mathrm{Al}$ concentration is increased from $6.1 \mathrm{wt} \%$ to $6.6 \mathrm{wt} \%$. (a), (b), and (c) are the $\mathrm{V}$ concentration while (d), (e), and (f) are the $\mathrm{Al}$ concentration.

Thus by using DFT in combination with cluster expansion theory and potential renormalization theory to calculate the local free energy, and incorporating with the phase field equation, we found clear element partitioning effects of $\mathrm{Al}$ and $\mathrm{V}$ in Ti64, i.e., a clear tendency that $\mathrm{Al}$ and $\mathrm{V}$ are selectively partitioned into $\alpha$ and $\beta$ phases, respectively, without using any thermodynamic parameter about the phases.

\section{Conclusion}

In this report, we have succeeded in extending the FPPF method to a ternary alloy, Ti64. Without using any thermodynamical parameter, element partitioning effects of $\mathrm{V}$ and $\mathrm{Al}$ in Ti64 were successfully investigated. The results clearly indicate the enrichment of $\mathrm{V}$ in the $\beta$ grain boundary while $\mathrm{Al}$ is concentrated in the $\alpha$ phase. By modifying the $\mathrm{V}$ and $\mathrm{Al}$ concentration by a very small amount, the transformation of the $\beta$ grain boundary and the $\alpha$ phase were also studied. All these results are in good agreements with recent experiments. Thus our FPPF theory is applicable not only to binary alloys but also to ternary alloys. We are planning to expand its application to more complicated systems, such as multielement alloys with $\mathrm{Al}$ and $\mathrm{Mg}$, to calculate the composition ratio dependency of the alloy and clarify each feature. This will make it possible to elucidate the essence of alloys that have been discussed only by empirical rules, and to realize the necessary properties such as strength, toughness, ductility, plasticity, and lightness to the maximum. 


\section{Acknowledgments}

This work has been supported by the grant-in-aid for Scientific Research on Innovative Areas (grant no. 25104713) from MEXT. We have been indebted to the HPCI social and scientific priority issue "Creation of new functional devices and high-performance materials to support next-generation industries" to be tackled by using post-K computer promoted by MEXT for the use of the supercomputer facilities at the Institute for Solid State Physics, the University of Tokyo, at Hokkaido University, and at the Institute for Materials Research, Tohoku University (Project IDs. hp180125, hp180220, and hp190180).

\section{Appendix}

We have developed a protocol, which can automatically generate and submit a huge number of FP calculations. DFT [26,27], cluster expansion theory [28] and potential renormalization theory $[29,30]$ were applied for setting up the calculation in this protocol. The outline picture of the protocol is presented in Fig.7. The input parameters are as follows. For the type of element, currently it can be set to calculate up to ternary systems. There are 3 lattice types: HCP, BCC, and FCC. The lattice parameters can be set by using the experimental data or the optimization data result of lattice optimization for primitive systems. The supercell size is set as $2 \times 2 \times 2$, and temperature can be set at any value. DFT calculation is performed using CASTEP program based

on GGA. With the help of this Pipeline Pilot protocol, the computational cost for the DFT calculations is significantly reduced. 


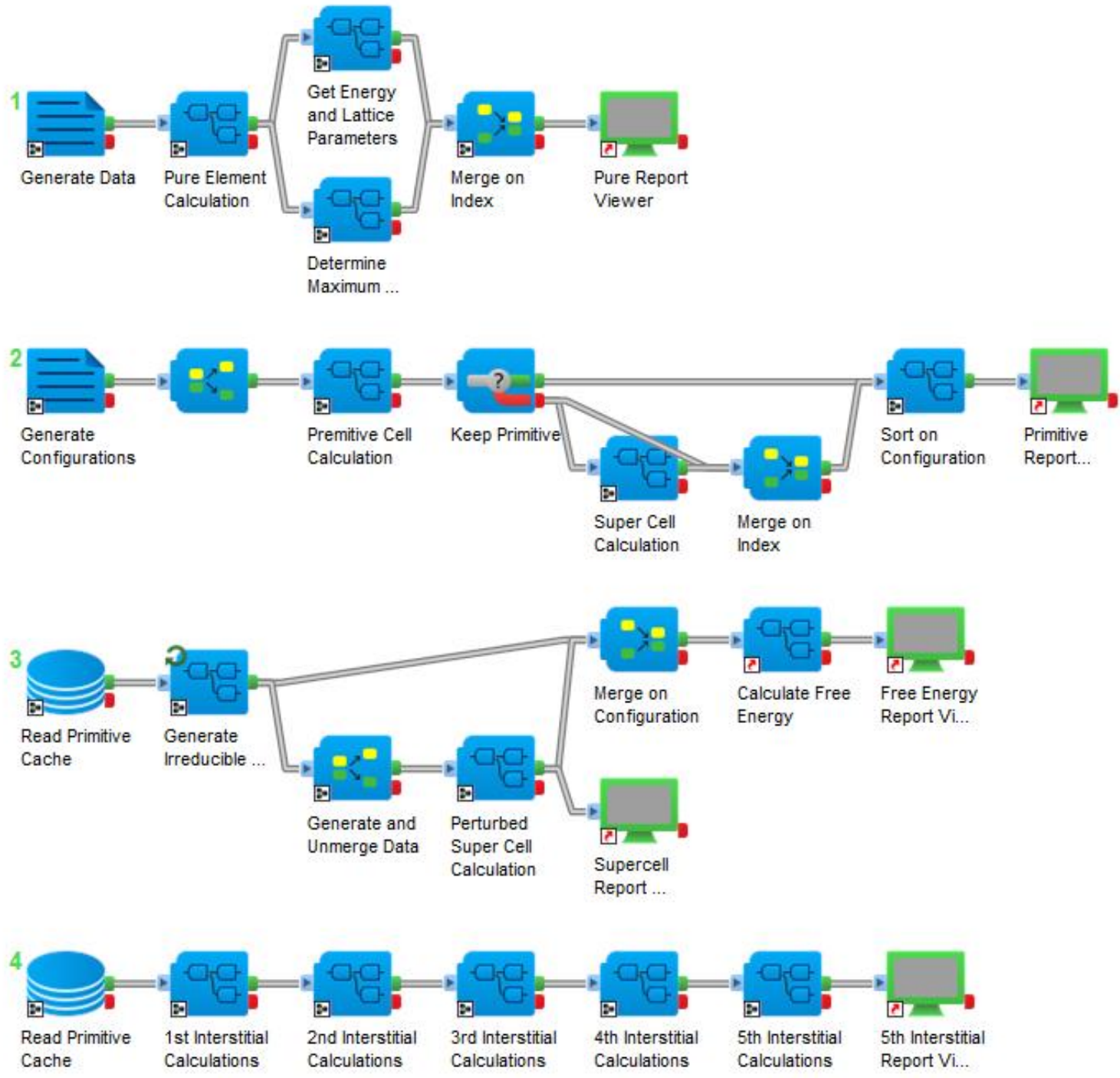

Figure 7: The Pipeline Pilot protocol has 5 main steps of calculations: for pure system, primitive system, super cell, free energy and interstitial.

\section{References}

[1] T. Seshacharyulu, S. C. Medeiros, W. G. Frazier, and Y. V. R. K. Prasad. Microstructural mechanisms during hot working of commercial grade ti-6al-4v with lamellar starting structure. Materials Science and Engineering: A, 325(1):112 - 125, 2002.

[2] R. Boyer, G. Welsch, and E. W. Collings. Materials Properties Handbook: Titanium Alloys. ASM International, 1994.

[3] S. M. C. van Bohemen, A. Kamp, R. H. Petrov, L. A. I. Kestens, and J. Sietsma. Nucleation and variant selection of secondary $\alpha$ plates in a $\beta$ ti alloy. Acta Materialia, 56(20):5907 - 5914, 2008.

[4] D. Wang, R. Shi, Y. Zheng, R. Banerjee, H. L. Fraser, and Y. Wang. Integrated computational materials engineering (icme) approach to design of novel microstructures for ti-alloys. JOM, 66(7):1287-1298, May 2014.

[5] S. L. Semiatin, T. M. Brown, T. A. Goff, R. E. Fagin, P. N.and Turner, J. M. Murry, D. R. Barker, 
J. D. Miller, and F. Zhang. Diffusion coefficients for modeling the heat treatment of ti-6al-4v. Metallurgical and Materials Transactions A, 35(9):3015-3018, Sep 2004.

[6] R. Ding and Z. X. Guo. Microstructural evoluion of a ti-6al-4v alloy during beta-phase processing: experimental and simulative investigations. Materials Science and Engineering: A, 365:172-179, Jan 2004.

[7] W. Sha and S. Malinov. Titanium Alloys: Modelling of Microstructure, Properties and Applications. Woohead Publishing Series in Metals and Surface Engineering. Elsevier, 2009.

[8] G. Lütjering. Influence of processing on microstructure and mechanical properties of $(\alpha+\beta)$ titanium alloys. Materials Science and Engineering: A, 243(1):32 - 45, 1998.

[9] S. Huang, Y. Ma, J. Qiu, H. Wang, J. Lei, B. Y. Zong, and R. Yang. Enhanced ambient temperature creep resistance of $\alpha / \beta$-ti alloys induced by minor fe. Materials Science and Engineering: A, 705:169-175, 2017.

[10] T. Li, M. Ahmed, G. Sha, R. Shi, G. Casillas, H.-W. Yen, Y. Wang, E. V. Pereloma, and J. M. Cairney. The influence of partitioning on the growth of intragranular $\alpha$ in near- $\beta$ ti alloys. Journal of Alloys and Compounds, 643:212 - 222, 2015.

[11] P. Mengucci, A. Gatto, E. Bassoli, L. Denti, F. Fiori, E. Girardin, P. Bastianoni, B. Rutkowski, A. Czyrska-Filemonowicz, and G. Barucca. Effects of build orientation and element partitioning on microstructure and mechanical properties of biomedical ti-6al-4v alloy produced by laser sintering. Journal of the Mechanical Behavior of Biomedical Materials, 71:1 - 9, 2017.

[12] X. Gao, W. Zeng, S. Zhang, and Q. Wang. A study of epitaxial growth behaviors of equiaxed alpha phase at different cooling rates in near alpha titanium alloy. Acta Materialia, 122:298 309, 2017.

[13] S. Huang, J. Zhang, Y. Ma, S. Zhang, S. S. Youssef, M. Qi, H. Wang, J. Qiu, D. Xu, J.Lei, and R. Yang. Influence of thermal treatment on element partitioning in $(\alpha+\beta)$ titanium alloy. Journal of Alloys and Compounds, 791:575 - 585, 2019.

[14] R. Banerjee, D. Bhattacharyya, P.C Collins, G.B Viswanathan, and H. L. Fraser. Precipitation of grain boundary $\alpha$ in a laser deposited compositionally graded ti-8al-xv alloy - an orientation microscopy study. Acta Materialia, 52(2):377 - 385, 2004.

[15] H. Tan, F. Zhang, J. Chen, X. Lin, and W. Huang. Microstructure evolution of laser solid forming of ti-al-v ternary system alloys from blended elemental powders. Chin. Opt. Lett., 9(5):051403, May 2011.

[16] J. Zhang, X. Li, D. Xu, and R. Yang. Recent progress in the simulation of microstructure evolution in titanium alloys. Progress in Natural Science: Materials International, 29:295-304, May 2019.

[17] R. Ding and Z. X. Guo. Coupled quantitative simulation of microstructural evolution and plastic flow during dynamic recrystallization. Acta Materialia, 49:3163-3175, Sep 2001.

[18] M. P. Anderson, G.S. Grest, and D. J. Srolovitz. Grain growth in three dimensions: A lattice model. Scripta Metallurgica, 19(2):225 - 230, 1985.

[19] A. D. Rollett, D. J. Srolovitz, R. D. Doherty, and M. P. Anderson. Computer simulation of recrystallization in non-uniformly deformed metals. Acta Metallurgica, 37(2):627 - 639, 1989.

[20] A. A. Wheeler, W. J. Boettinger, and G. B. McFadden. Phase-field model for isothermal phase transitions in binary alloys. Phys. Rev. A, 45:7424-7439, May 1992.

[21] L.-Q. Chen. Phase-field models for microstructure evolution. Annual Review of Materials Research, 32(1):113-140, Aug 2002.

[22] I. Steinbach. Phase-field model for microstructure evolution at the mesoscopic scale. Annual Review of Materials Research, 43(1):89-107, Jul 2013.

[23] F. Roters, P. Eisenlohr, L. Hantcherli, D. D. Tjahjanto, T. R. Bieler, and D. Raabe. Overview of constitutive laws, kinematics, homogenization and multiscale methods in crystal plasticity finiteelement modeling: Theory, experiments, applications. Acta Materialia, 58(4):1152 - 1211, 2010.

[24] D. Raabe and F. Roters. Using texture components in crystal plasticity finite element simulations. International Journal of Plasticity, 20(3):339 - 361, 2004. Owen Richmond Memorial Special Issue. 
[25] S. Bhattacharyya, R. Sahara, and K. Ohno. A first-principles phase field method for quantitatively predicting multi-composition phase separation without thermodynamic empirical parameter. Nature Communications, 10(1):3451, 2019.

[26] P. Hohenberg and W. Kohn. Inhomogeneous electron gas. Phys. Rev., 136:B864-B871, Nov 1964.

[27] W. Kohn and L. J. Sham. Self-consistent equations including exchange and correlation effects. Phys. Rev., 140:A1133-A1138, Nov 1965.

[28] J. W. D. Connolly and A. R. Williams. Density-functional theory applied to phase transformations in transition-metal alloys. Phys. Rev. B, 27:5169-5172, Apr 1983.

[29] K. Ohno. Renormalization of interatomic potentials and lattice gas models. Sci. Rep. Res. Inst. Tohoku Univ. A, 43:17, 1997.

[30] K. Ohno, K. Esfarjani, and Y. Kawazoe. Computational Materials Science: From Ab Initio to Monte Carlo Methods. Springer Series in Solid-State Sciences. Springer Berlin Heidelberg, 1999.

[31] G. D. Garbulsky and G. Ceder. Linear-programming method for obtaining effective cluster interactions in alloys from total-energy calculations: Application to the fcc pd-v system. Phys. Rev. B, 51:67-72, Jan 1995.

[32] G. Ceder, G. D. Garbulsky, and P. D. Tepesch. Convergent real-space cluster expansion for configurational disorder in ionic systems. Phys. Rev. B, 51:11257-11261, May 1995.

[33] S. J. Clark, M. D. Segall, C. J. Pickard, P. J. Hasnip, M. I. J. Probert, K. Refson, and M. C. Payne. First principles methods using castep. Zeitschrift für Kristallographie - Crystalline Materials, 220:567?570, 2009.

[34] J. P. Perdew. Generalized gradient approximation made simple. Physical Review Letters, 77(18):3865-3868, 1996.

[35] C. Leyens and M. Peters. Titanium and Titanium Alloys: Fundamentals and Applications. Wiley, 2003.

[36] Y.-L. Song, Z.-H. Dou, T.-A. Zhang, Y. Liu, and G.-C. Wang. First-principles calculation on the structural, elastic and thermodynamic properties of ti-al intermetallics. Materials Research Express, 6(10):1065a4, Sep 2019.

[37] P.-Y. Tang, B.-Y. Tang, and X.-P. Su. First-principles studies of typical long-period superstructures al5ti3, h-al2ti and r-al2ti in al-rich tial alloys. Computational Materials Science, 50(4):1467 - 1476, 2011. Proceedings of the 19th International Workshop on Computational Mechanics of Materials.

[38] T. Hong, T. J. Watson-Yang, X.-Q. Guo, A. J. Freeman, T. Oguchi, and J.-H Xu. Crystal structure, phase stability, and electronic structure of ti-al intermetallics: ti $i_{3}$ al. Phys. Rev. B, 43:1940-1947, Jan 1991.

[39] N. Zheng and Y. Jin. Band-gap and slater-pauling rule in half-metallic ti2-based heusler alloys: A first-principles study. Journal of Magnetism and Magnetic Materials, 324(19):3099 - 3104, 2012.

[40] G. G. E. Seward, S. Celotto, D. J. Prior, J. Wheeler, and R. C. Pond. In situ sem-ebsd observations of the hcp to bcc phase transformation in commercially pure titanium. Acta Materialia, $52(4): 821-832,2004$.

[41] S. R. Nishitani, H. Kawabe, and M. Aoki. First-principles calculations on bcc-hcp transition of titanium. Materials Science and Engineering: A, 312(1):77-83, 2001.

[42] E. Alabort, P. Kontis, D. Barba, K. Dragnevski, and R. C. Reed. On the mechanisms of superplasticity in ti-6al-4v. Acta Materialia, 105:449 - 463, 2016. 